\title{
A data-based comparison of flood frequency analysis methods used in France
}

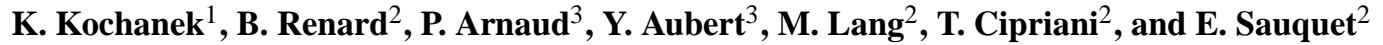 \\ ${ }^{1}$ Institute of Geophysics, Polish Academy of Sciences, Księcia Janusza 64, 01-452, Warsaw, Poland \\ ${ }^{2}$ Irstea Lyon, UR HHLY Hydrology-Hydraulics, 5 rue de la Doua CS70077, 69626 Villeurbanne CEDEX, France \\ ${ }^{3}$ Irstea Aix-En-Provence UR OHAX, 3275 Route de Cézanne, CS 40061, 13182 Aix-en-Provence CEDEX 5, France
}

Correspondence to: K. Kochanek (kochanek@igf.edu.pl)

Received: 31 July 2013 - Published in Nat. Hazards Earth Syst. Sci. Discuss.: 6 September 2013

Revised: 8 January 2014 - Accepted: 10 January 2014 - Published: 20 February 2014

\begin{abstract}
Flood frequency analysis (FFA) aims at estimating quantiles with large return periods for an extreme discharge variable. Many FFA implementations are used in operational practice in France. These implementations range from the estimation of a pre-specified distribution to continuous simulation approaches using a rainfall simulator coupled with a rainfall-runoff model. This diversity of approaches raises questions regarding the limits of each implementation and calls for a nation-wide comparison of their predictive performances.

This paper presents the results of a national comparison of the main FFA implementations used in France. More accurately, eight implementations are considered, corresponding to the local, regional and local-regional estimation of Gumbel and Generalized Extreme Value (GEV) distributions, as well as the local and regional versions of a continuous simulation approach. A data-based comparison framework is applied to these eight competitors to evaluate their predictive performances in terms of reliability and stability, using daily flow data from more than 1000 gauging stations in France.

Results from this comparative exercise suggest that two implementations dominate their competitors in terms of predictive performances, namely the local version of the continuous simulation approach and the local-regional estimation of a GEV distribution. More specific conclusions include the following: (i) the Gumbel distribution is not suitable for Mediterranean catchments, since this distribution demonstrably leads to an underestimation of flood quantiles; (ii) the local estimation of a GEV distribution is not recommended, because the difficulty in estimating the shape parameter results in frequent predictive failures; (iii) all the purely regional
\end{abstract}

implementations evaluated in this study displayed a quite poor reliability, suggesting that prediction in completely ungauged catchments remains a challenge.

\section{Introduction}

\subsection{Diversity of flood frequency analysis approaches}

The flood frequency analysis (FFA) is a central step in a hydrological risk assessment. In general the FFA aims at estimating "flood quantiles", i.e., discharge values whose return period is large (usually $>10$ years). It has many operational applications, including design of civil engineering structures (e.g., polders, bridges, levees, dam spillways, protection structures for nuclear power plants) or mapping of flood-prone areas. Many FFA approaches exist in the literature. In France, two distinct families of approach are used in practice. The first family comprises FFA implementations that estimate the parameters of a given flood distribution (a Gumbel or a Generalized Extreme Value (GEV) distribution in most cases). The second family uses a continuous simulation approach (Arnaud and Lavabre, 1999, 2002), where a rainfall generator is coupled with a rainfall-runoff model to generate long hydrological series from which extreme quantiles can be inferred. Within both families, parameter estimation can be performed at the local scale using at-site streamflow data only (e.g., Kuczera, 1999; Martins and Stedinger, 2000), at the regional scale using streamflow data from neighboring stations only (e.g., Stedinger and Tasker, 
1985, 1986; Hosking and Wallis, 1997), or combining local and regional information (e.g., Ribatet et al., 2006).

Many countries prepared and issued national FFA guidelines to help practitioners in realizing their analyses with best practice methods, e.g., (Reed et al., 1999; Institution of Engineers Australia, 1987; Interagency Advisory Committee on Water Data, 1982; Stewart et al., 2008). This is not the case in France, where no specific FFA implementation is officially recommended, let alone prescribed by regulation. While practitioner-oriented documents describing the main approaches to FFA have been published (Lang et al., 2007), an extensive comparison of the main FFA implementations used in operational practice in France remains to be performed.

\subsection{Challenges facing the evaluation and comparison of FFA approaches}

A large number of comparative studies of FFA implementations have been reported in the research literature (e.g., Hosking et al., 1985; Gunasekara and Cunnane, 1992; Kroll and Stedinger, 1996; GREHYS, 1996; Ouarda et al., 2006; Meshgi and Khalili, 2009; Sankarasubramanian and Srinivasan, 1999). The comparison framework varies from one study to another, and can be based on Monte Carlo simulations, statistical tests, graphical methods and so on. Bobee et al. (1993) therefore advocated "a systematic approach to comparing distributions used in flood frequency analysis", which is still not agreed upon to our best knowledge.

In the context of the present paper, where distinct FFA families are to be compared, the comparison framework can hardly be based on Monte Carlo simulations. Indeed, this would require setting up a synthetic experiment to generate "true" data that can be used by all FFA implementations. Ensuring a "fair" simulation setup that would not advantage a particular FFA implementation is feasible when similar implementations are considered (e.g., comparing several local estimation methods for a given distribution). However, it is more difficult when both local and regional estimation approaches are considered: how to realistically simulate spatially dependent extremes on a river network? What is a realistic misspecification of the regression model used in regional approaches? Ensuring the fairness of the simulation setup is even more challenging if continuous simulation approaches are considered (How to realistically simulate the non-linearity of the rainfall-runoff relationship? How to simulate realistic structural errors for the rainfall simulator or the hydrologic model?).

An alternative to Monte Carlo comparisons is to implement data-based predictive comparisons, where the estimations from all competing FFA implementations are simply compared with validation data (Gunasekara and Cunnane, 1992; Interagency Advisory Committee on Water Data, 1982). Recently, Renard et al. (2013) proposed a data-based comparison framework that could be applied to any FFA implementation. This framework complements (but not replaces) alternative comparison methods based, for instance, on Monte Carlo simulations. Most important, this framework enables the comparison of any FFA implementation belonging to each family presented in Sect. 1.1.

\subsection{Objectives of the paper}

In this paper we present the results of a nation-wide comparison of the predictive performances of FFA implementations in order to find the limits of each implementation and, if possible, recommend the best FFA methodology for the French rivers. By best methodology we mean best performance of a particular implementation according to the indices described later in Sect. 2.3.

The paper is organized as follows. Section 2 presents the data and methods used in this paper, including the competing FFA implementations (Sect. 2.2), a summary of the comparison framework (Sect. 2.3) and the comparison data set (Sect. 2.4). Section 3 describes the main results of the comparison, and Sect. 4 further discusses them. Conclusions are drawn in Sect. 5.

\section{Data and methods}

\subsection{Short description of the ExtraFlo project}

By their nature and danger, the extreme floods cannot be observed often and thoroughly enough to collect the sample adequate for further statistical analysis. Instead, the probabilistic study of extreme values relies on the analysis of a limited (in number and space) series of events in time, to infer a probabilistic behavior of a particular case which is then extrapolated to the whole population of floods. This procedure encounter certain difficulties:

- How to extrapolate excessively short time series, when information is generally available on more or less recent events.

- How to set design values for the whole of the territory, whereas the density of the measurement is inevitably limited owing to the spatial variability of rainfall and discharge data.

- How to update our knowledge of extreme events in a non-stationary context.

The objective of the ExtraFlo project (Extreme Rainfall and Floods) was to carry out an inter-comparison of the methods for estimating extreme rainfall and floods used in France, to obtain a better understanding of their respective fields of application. FFA has a wide variety of applications in France: small return periods ( $\sim 10$ years) are sometimes sufficient for secondary infrastructures or for disaster declarations. Hazard mapping typically uses 100-year return periods, while 
some civil engineering structures (large dams, nuclear power plants) may require $10^{3}-10^{4}$ target return periods.

A particular emphasis was placed during the project on compiling reference data sets (long at-site time series and regional sets) to pinpoint the pros and cons of each approach with their sensitivity to the increasing information. As a result of this work methods for estimating extreme values of floods were developed and improved. Moreover, the project placed a range of practical tools concerning the management of flood risk that can be estimated not only according to the hydrological characteristics of river basins but also on the basis of available information.

\subsection{Competing teams: FFA implementations}

Eight implementations that are frequently used in France are compared in this paper. Note that other approaches are also used in operational practice, in particular the SCHADEX semi-continuous simulation approach (Paquet et al., 2006) or the SPEED method (Cayla, 1995). However they could not be included in this comparative exercise because they could not be fully automated and were therefore not suitable for an application to thousands of sites (see Sect. 2.4). The eight competing implementations are split according to their estimation scale: purely local, purely regional or mixed localregional.

\subsubsection{The local league: using at-site runoff data only}

The local league contains the following three implementations:

1. Implementation LOC_GUM, corresponding to the estimation of a two-parameter Gumbel distribution using at-site annual maxima (see Sect. AA for details on the Gumbel distribution). A Bayesian estimation is performed, with flat priors $(\pi(\theta) \propto 1)$ for both the location and the scale parameters. Maximum-posterior values are used as parameter estimates.

2. Implementation LOC_GEV, corresponding to the estimation of a three-parameter GEV distribution using at-site annual maxima (see Sect. AA for details on the GEV distribution). A Bayesian estimation is also performed, with flat priors $(\pi(\theta) \propto 1)$ for the location and the scale parameters, and a Gaussian prior with mean zero and standard deviation 0.25 for the shape parameter.

3. Implementation LOC_SHY is the local version of the SHYREG method, a continuous simulation approach, coupling a rainfall generator with a rainfall-runoff model to estimate flood quantiles. A description of the various versions of SHYREG can be found in Arnaud and Lavabre (2002), Aubert (2013) and Organde et al. (2013).
For the first two implementations, the Gumbel and GEV distributions are chosen because they are the most widely used methods in hydrological projects in France (despite the fact that there is no prescribed distribution officially recommended for FFA in France). Moreover, preliminary analyses (not shown) indicated that they performed at least equally well as other distributions, including Log-Normal, Pearson III or Log-Pearson III distributions. The choice of the Bayesian estimation approach is made to facilitate the use of a unique estimation approach at local, regional and localregional scales. Preliminary analyses (Kochanek et al., 2012; Renard et al., 2013) show that for a given record length, the impact of the estimation approach (e.g., Bayesian, maximum likelihood, moments, linear moments) is small compared to the choice of the parent distribution or the choice of the estimation scale (local, regional or local-regional). The analysis was carried out for the same record length $(N)$ for the competing implementations (see Sect. 2.4 for details).

Regarding the third implementation, note that LOC_SHY uses a regionalized version of the rainfall generator, but is local with respect to discharge data in the sense that the rainfall-runoff model is estimated with local data. The regionalization of the rainfall generator is based on a data set comprising 2812 daily rain gauges with at least 20 years of data over the period 1977-2002 (Arnaud et al., 2008). Regionalization is performed for the three parameters of the rainfall generator, namely: the average number of storms, the average storm intensity and the average storm duration. Regionalized parameters are then available on a $1 \times 1 \mathrm{~km}$ grid. The fact that these parameters represent averages over many storms (average number, intensity and duration) induces a much lesser sensitivity to sampling variability than more extreme characteristics (see Carreau et al., 2013).

\subsubsection{The regional league: estimation in ungauged catchments}

The regional league contains the following three implementations:

1. Implementation REG_GUM, corresponding to the regional estimation of a Gumbel distribution by means of regressions linking its parameters with catchment characteristics.

2. Implementation REG_GEV, corresponding to the regional estimation of a GEV distribution.

3. Implementation REG_SHY, the fully regionalized version of the continuous simulation implementation SHYREG (Aubert, 2013; Organde et al., 2013).

Section AA1 provides additional details on the regionalization procedures for the first two implementations. Again, the choice of these particular two implementations is based on preliminary analyses (Cipriani et al., 2012) that are not fully described here. 
The third implementation REG_SHY uses the same regionalized rainfall generator as implementation LOC_SHY. However, unlike LOC_SHY, it uses a regionalized version of the rainfall-runoff model. The details of this regionalization procedure are given by Organde et al. (2013). In the current paper, the regionalization procedure is re-implemented based on the runoff data set used to compare the FFA implementations (see Sect. 2.4). Since most of the runoff data set covers the period 1969-2011, the regionalization of the rainfallrunoff model is based on a period that encompasses the period used for regionalizing the rainfall generator (1977-2002, see Sect. 2.2.1).

\subsubsection{The local-regional league: combining at-site data and regional information}

The local-regional league contains the following two implementations:

1. Implementation L+R_GUM, corresponding to the local-regional estimation of a Gumbel distribution, using both local and regional information.

2. Implementation L+R_GEV, corresponding to the local-regional estimation of a GEV distribution.

The combination of local and regional information is straightforward in the Bayesian context adopted here: regional estimates are used to specify a prior distribution, while local data are used to compute the likelihood function (see Sect. A2 for additional details).

\subsection{Referees: stability and reliability indices}

The evaluation criteria are based on performance indices quantifying the reliability and the stability of the FFA implementations. A complete description of the motivation behind these performance indices is given in Renard et al. (2013). In this paper we therefore restrict ourselves to a short presentation of these indices and their practical use to compare FFA implementations.

\subsubsection{Reliability indices}

The reliability indices aim to evaluate the agreement between the estimated distribution at site $i$ (whose cumulative distribution function, CDF, is noted $\hat{F}^{(i)}$ ) and validation observations $\left(d_{k}^{(i)}\right)_{k=1: n^{(i)}}$. Importantly, this requires splitting available data into calibration and validation subsamples. The decomposition adopted in this paper will be described in Sect. 2.4.

The first reliability index was used, e.g., by England et al. (2003) and Garavaglia et al. (2011), and corresponds to the CDF of the estimated distribution computed on the largest validation data. For a given site $i$, it is computed as follows:

$F F^{(i)}=\hat{F}^{(i)}\left(d_{\max }^{(i)}\right)$.
If the estimation is reliable (i.e., $\hat{F}^{(i)}=F^{(i)}$, where $F^{(i)}$ denotes the unknown true CDF), it can be shown (Renard et al., 2013) that $F F^{(i)}$ is a realization from a Beta distribution with parameters $\left(n^{(i)} ; 1\right): F F^{(i)} \sim \operatorname{Beta}\left(n^{(i)} ; 1\right)$, whose $\mathrm{CDF} F_{\text {Beta }}$ can be written as $F_{\text {Beta }}(t)=t^{n^{(i)}}, 0 \leq t \leq 1$. The $F F$ index focuses on the right tail of the estimated CDF by using the largest element in the validation sample. The adequacy between $F F^{(i)}$ values observed over all validation sites and their theoretical Beta distributions under the reliability hypothesis will be assessed using graphical diagnostics and reliability scores that will be described in Secs. 2.3.3. and 2.3.4.

The second reliability index is based on the number of exceedances (within the validation sample) of an estimated $T$-year quantile $\hat{q}_{T}^{(i)}$ (e.g., Interagency Advisory Committee on Water Data, 1982; Gunasekara and Cunnane, 1992; Garavaglia et al., 2011):

$N_{T}^{(i)}=\sum_{k=1}^{n^{(i)}} 1_{\left(\hat{q}_{T}^{(i)} ;+\infty\right)}\left(d_{k}^{(i)}\right), \quad$ where $1_{A}(x)=\left\{\begin{array}{c}1 \text { if } x \in A \\ 0 \text { otherwise }\end{array}\right.$.

Under the reliability assumption $\left(\hat{q}_{T}^{(i)}=q_{T}^{(i)}\right), N_{T}^{(i)}$ is a realization from the binomial distribution:

$N_{T}^{(i)} \sim \operatorname{Bin}\left(n^{(i)}, 1 / T\right)$

(Renard et al., 2013). The $N_{T}$ index focuses on reliability for prescribed $T$-year quantiles. In this paper, we are going to analyze $N_{T=10}$ and $N_{T=100}$ values for 10-year and 100-year floods, respectively.

\subsubsection{Stability index}

The stability of quantile estimates can be quantified by contrasting the values obtained with two different calibration data sets $\boldsymbol{c}_{1}$ and $\boldsymbol{c}_{2}$. The decomposition into two calibration subsamples adopted in this paper will be described in Sect. 2.4. We stress that the stability is only a secondary consideration compared to reliability: indeed, a FFA implementation can be totally unreliable but perfectly stable. Consequently, stability is seen as an additional quality used to further discriminate FFA implementations that would have similar reliability.

The index $\operatorname{SPAN}_{T}$ (Garavaglia et al., 2011) used in this paper is a measure of the span of two $T$-year quantiles estimated with distinct calibration data sets. For a given site $i$, it is defined as follows:

$\operatorname{SPAN}_{T}^{(i)}=2 \frac{\left|\hat{q}_{T}^{(i)}\left(\boldsymbol{c}_{1}\right)-\hat{q}_{T}^{(i)}\left(\boldsymbol{c}_{2}\right)\right|}{\hat{q}_{T}^{(i)}\left(\boldsymbol{c}_{1}\right)+\hat{q}_{T}^{(i)}\left(\boldsymbol{c}_{2}\right)}$.

The FFA implementation whose $\mathrm{SPAN}_{T}$ is closest to zero is the most stable. 

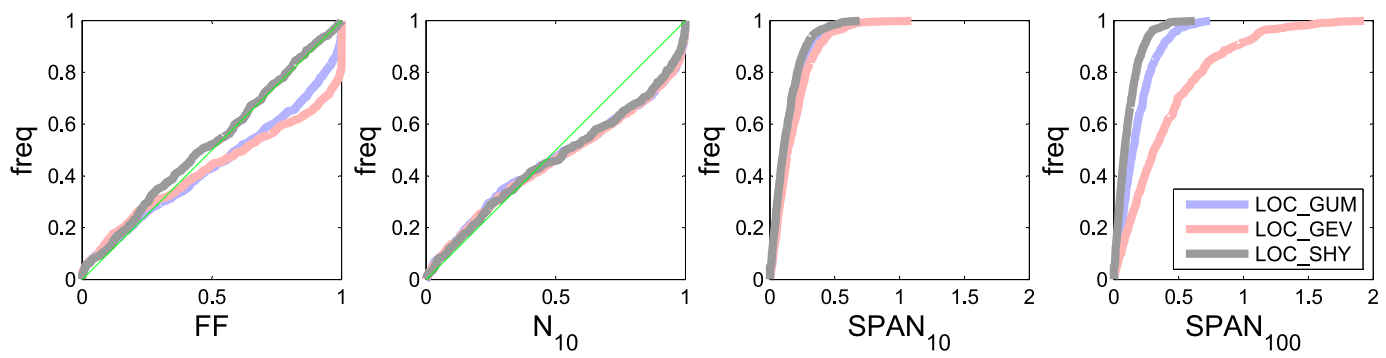

Fig. 1. Reliability $\left(F F, N_{10}\right)$ and stability $\left(\mathrm{SPAN}_{10}, \mathrm{SPAN}_{100}\right)$ indices for local implementations.

\subsubsection{Graphical representations}

\section{Reliability}

Graphical representation of reliability is based on the comparison between the calculated indices and their theoretical distribution under the reliability assumption. Note that for both reliability indices, this theoretical distribution depends on the number of validation observations $n^{(i)}$, that may vary from site to site. To circumvent this problem, a probabilityprobability plot (pp-plot) representation is adopted: raw indices are transformed into probabilities by applying the CDF of their theoretical distribution under the reliability hypothesis. Under the reliability hypothesis, the probabilitytransformed values are then uniformly distributed between 0 and 1 , regardless of the sample size $n^{(i)}$. It is therefore possible to plot the probability-transformed values for all sites against empirical frequencies, yielding reliability pp-plots as illustrated in Fig. 1. Curves closer to the diagonal correspond to more reliable FFA implementations.

Note that for the $N_{T}$ index, the theoretical binomial distribution is a discrete distribution. It is therefore necessary to randomize its probability-transformed values in order to ensure that they are uniformly distributed. The randomization procedure is described in Renard et al. (2013).

\section{Stability}

The comparison of stability between competing FFA implementations is based on comparing the distribution of $\operatorname{SPAN}_{T}^{(i)}$ over all sites $i=1: N_{\text {sites }}$, as illustrated in Fig. 1. The FFA implementation whose $\mathrm{SPAN}_{T}$ distribution remains the closest to zero is the most stable.

\subsubsection{Scores}

The graphical representations can be further summarized into numerical scores that will provide a more synthetic view of the performances of FFA implementations over the various performance indices.
For reliability indices $F F$ and $N_{T}$, the score is based on the area between the diagonal line and the reliability curve, with a normalization ensuring that the score is varying between 0 (low reliability) and 1 (perfect reliability). For any probability-transformed index $w$, the score can be computed as

$$
\begin{gathered}
\text { score }=1-2 \cdot \text { Area }(\text { curve }, \text { diagonal })= \\
=1-\frac{2}{N_{\text {site }}+1} \sum_{i=1}^{N_{\text {site }}}\left|w^{(i)}-\frac{i-0.5}{N_{\text {site }}}\right| .
\end{gathered}
$$

Analogically, a stability score can be derived based on the area between the $y$ axis and the $\operatorname{SPAN}_{T}$ curve, normalized to vary between 0 (low stability) and 1 (perfect stability):

$$
\begin{aligned}
& \text { score }=1-0.5 \cdot \operatorname{Area}(\text { curve, } y \text { axis) } \\
& =1-\frac{1}{2 N_{\text {site }}} \sum_{i=1}^{N_{\text {site }}} \operatorname{SPAN}_{T}^{(i)} .
\end{aligned}
$$

\subsection{Playground: daily runoff data set}

\subsubsection{Data set description}

Daily runoff series from 1076 gauging stations located throughout France are used (see Fig. 2). Catchment sizes range from 10 to $2000 \mathrm{~km}^{2}$. All series have at least 20 years of data (20-39 years: 535 stations ( $49.7 \%)$; 40-59 years: 476 stations $(44.2 \%)$; 60 years and more: 65 stations $(6.1 \%)$ ). The quality-control procedures have been implemented to remove stations with measurement problems or stations corresponding to heavily regulated catchments. This data set is therefore an extension of the data set used by Renard et al. (2008), including updated series (until 2012) and many more stations.

These 1076 gauging stations are representative of the main hydrological and climate regimes found in mainland France. For regime-specific analyses, it is useful to cluster these stations into homogenous regions. This is achieved by using the hydroecoregions (HER) defined by Wasson et al. (2004), as illustrated in Fig. 2. These regions are based on topographic, geological and climate (precipitations and temperature) maps. Importantly, they do not directly use any runoff data, and can therefore be defined independently from the FFA analyses performed here. 


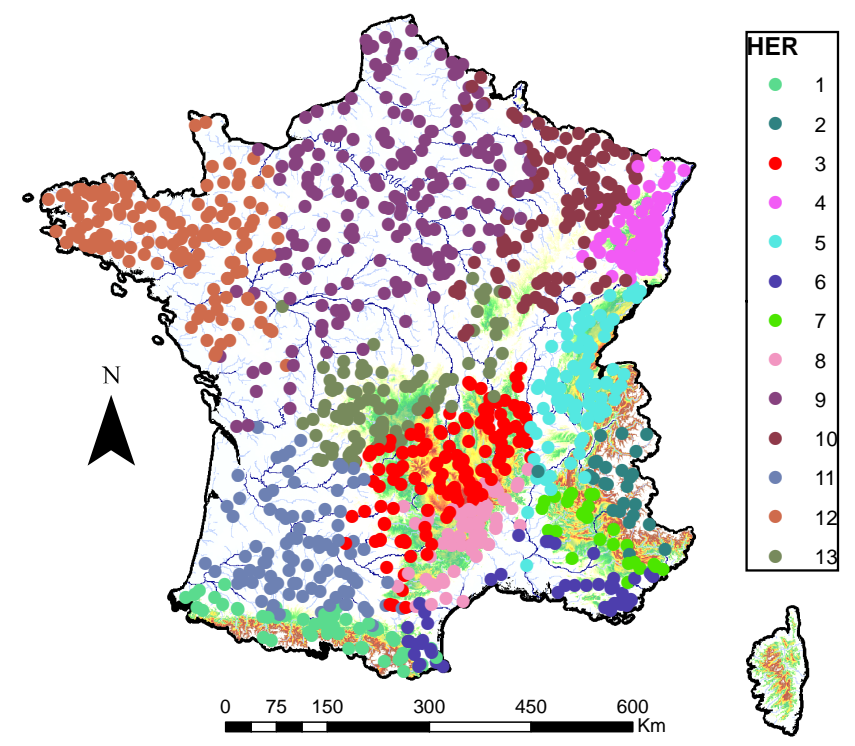

Fig. 2. Location of the gauging stations used in this study.

\subsubsection{Reliability and stability decompositions}

As explained in Sect. 2.3, the computation of reliability indices requires decomposition of all data set time series into calibration-validation subsamples. For stability the data sets are also divided into two subsamples: calibration data set no. $1\left(\boldsymbol{c}_{1}\right)$ and calibration data set no. $2\left(\boldsymbol{c}_{2}\right)$. These decompositions are performed as follows: for reliability, the 593 series with 20 to 40 years of data are used to calibrate regional implementations, or the regional part of local-regional implementations. The remaining 483 series (with more than 40 years of data) are further decomposed:

- 20 years are randomly chosen (independently on each site) to calibrate the local implementations, or the local part of local-regional implementations.

- All remaining years (at least 20 years) are used as validation data. Importantly, the validation data are therefore exactly the same for all implementations.

For stability, two distinct types of decompositions are implemented:

- The type I decomposition focuses on stability with respect to local data: for each of the 483 series with more than 40 years, 20 years are randomly assigned to the $c_{1}$ subsample, and 20 other years are randomly assigned to the $\boldsymbol{c}_{2}$ subsample. Obviously, purely regional implementations are insensitive to this decomposition, since they do not use local data.

- The type II decomposition focuses on stability with respect to regional data: the 593 series with 20 to
40 years of data are randomly split into two subsamples $\boldsymbol{c}_{1}$ and $\boldsymbol{c}_{2}$. Obviously, purely local implementations are insensitive to this decomposition, since they do not use regional data.

\section{Results}

\subsection{Comparison of quantile estimates}

Before describing the comparison in terms of reliability and stability, it is of interest to assess how different the various competing implementations are. To this aim, Fig. 3 compares the 100-year flood estimated by each implementation with the one estimated by the implementation LOC_GUM, considered as the reference in this figure.

Both local implementations LOC_GEV and LOC_SHY systematically yield larger quantiles in southeastern France (sometimes exceeding $+40 \%$ ). Elsewhere in the country, smaller and larger quantiles are found with no clear spatial pattern for implementation LOC_GEV, while LOC_SHY quantiles tend to be systematically larger than LOC_GUM ones.

The local-regional implementation L+R_GUM generally yields small differences with the reference, suggesting that for a Gumbel distribution, local and local-regional estimations yield similar estimates. By contrast, the local-regional implementation L+R_GEV yields markedly higher quantiles in southeastern France.

All three regional implementations REG_GUM, REG_GEV and REG_SHY yield marked differences (both positive and negative) with the reference, but no distinctive spatial pattern can be observed. This suggests that the estimation scale (local or regional) has an important impact on quantile estimates.

\subsection{Results for the local league}

Figure 1 shows reliability and stability indices for the local implementations. Amongst them, LOC_SHY clearly outperforms its two opponents (LOC_GUM and LOC_GEV): it is both more reliable (especially for extreme values, index $F F$ ) and more stable. The poor performance of the locally estimated GEV distribution is worth noting: it is markedly unreliable and much less stable than other implementations (especially for high quantiles). The behavior of the $F F$ curves near the upper-right corner is noteworthy: it indicates that for about $20 \%$ of the stations, a flood observed during the validation period was deemed impossible by LOC_GEV (yielding $F F$ values equal to one). This is due to errors in estimating the shape parameter of the GEV distribution, yielding an upper bound for the estimated GEV pdf that is exceeded during the validation period. Note that this does not imply that the GEV distribution should be avoided: the problem might rather be due to its local estimation, as will be further discussed in Sect. 3.4. 

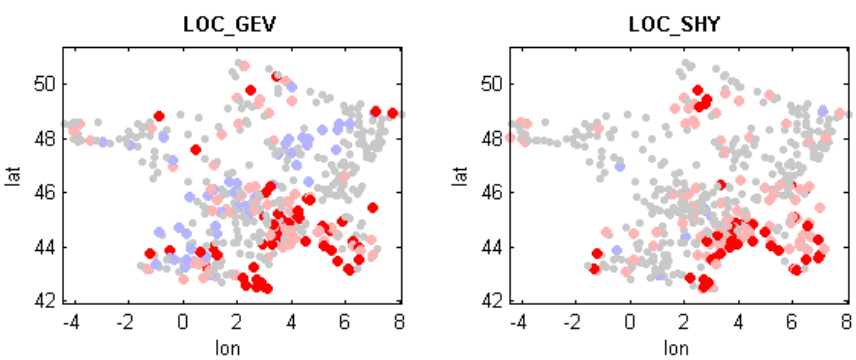

Difference with reference (LOC_GUM)
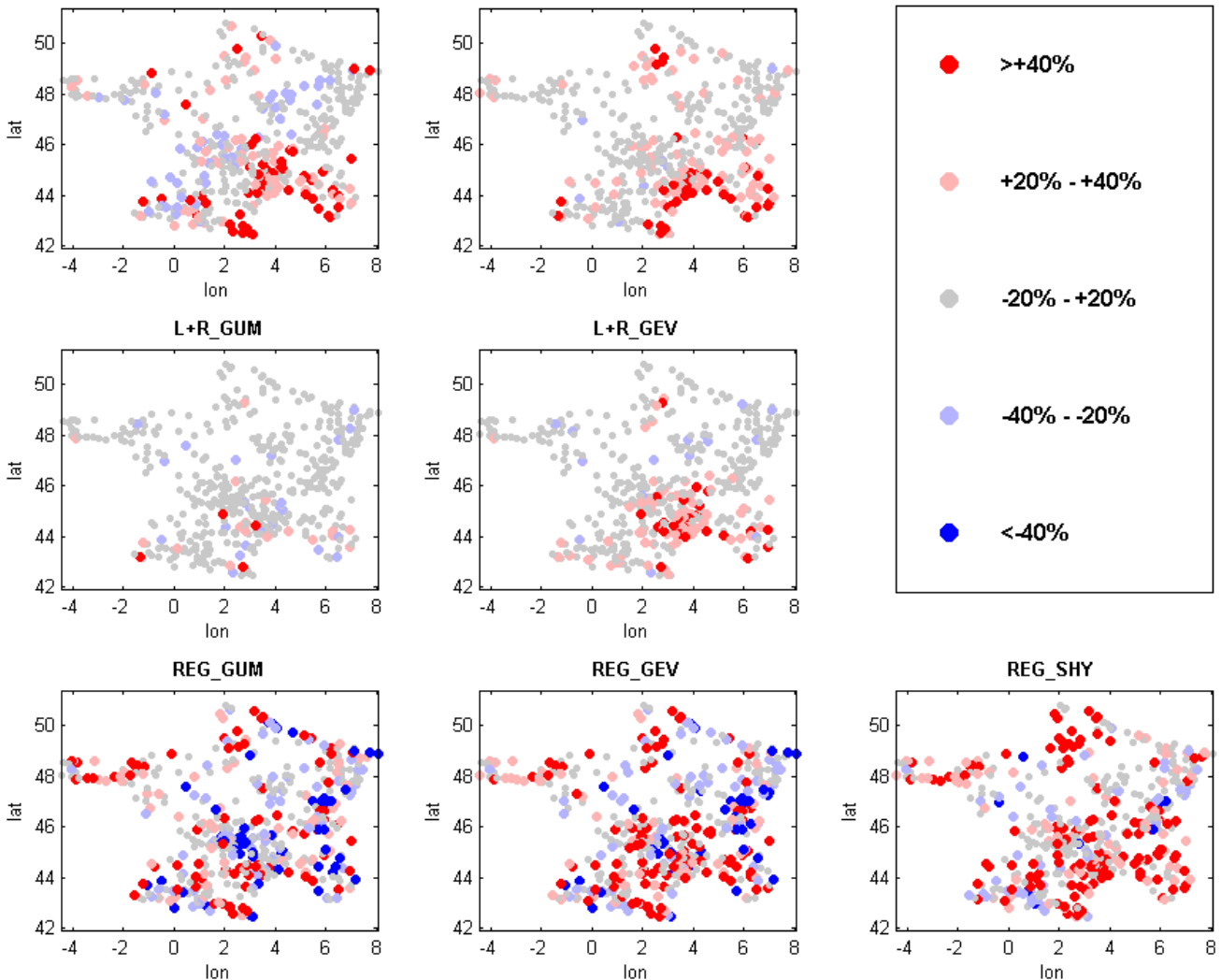

Fig. 3. Relative differences between 0.99 quantiles.
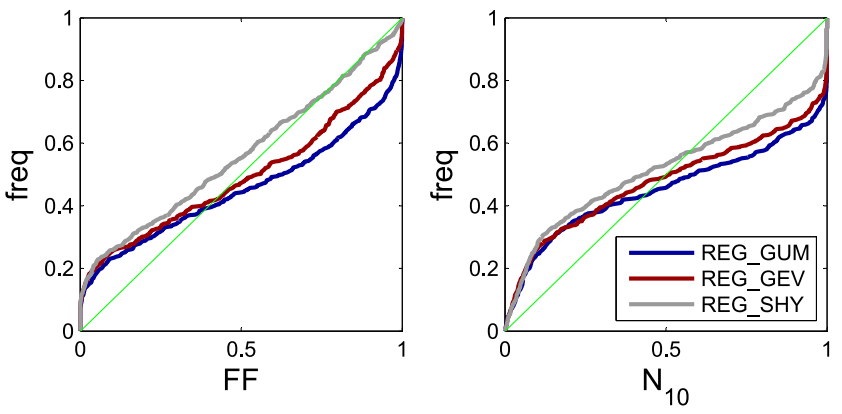

Fig. 4. Reliability indices for regional implementations.

\subsection{Results for the regional league}

Figure 4 shows reliability indices for regional implementations and shows that none of them reach an acceptable reliability. The continuous simulation implementation REG_SHY appears more reliable for index $F F$, but still yields unreliable predictions for the 10-year flood, as shown by index $N_{10}$. More detailed analyses (not shown here) suggest that the main reason for such poor performances is the difficulty in setting up a regression with catchments' characteristics: the explanatory power of such regressions remains quite low and result in unreliable predictions at ungauged sites.
Note that we omitted the results in terms of stability for the regional implementations. Indeed, as noted in Sect. 2.3.2, stability is only a secondary consideration (compared with reliability) and is used only to discriminate implementations that would be comparably reliable. In this particular case, reliability is poor for all implementations (see Fig. 4), so we decided that stability was not worth considering (a stable but non-reliable implementation being worthless).

\subsection{Results for the local-regional league}

Figure 5 shows reliability and stability indices for the two local-regional implementations for Gumbel and GEV distributions. Both implementations yield similar results: the reliability is acceptable and stabilities are similar. The use of a GEV distribution yields slightly more reliable predictions according to index $F F$, at the cost of a slightly lower stability with respect to regional information (type II). The differences between the Gumbel and GEV distributions will be further discussed in Sect. 3.5.

It is important to notice that the local-regional estimation of a GEV distribution yields acceptable reliability and stability, which was not the case for purely local or regional estimation approaches. This is illustrated in Fig. 6, which compares those three estimation approaches. In terms of reliability, implementation L+R_GEV clearly outperforms its 

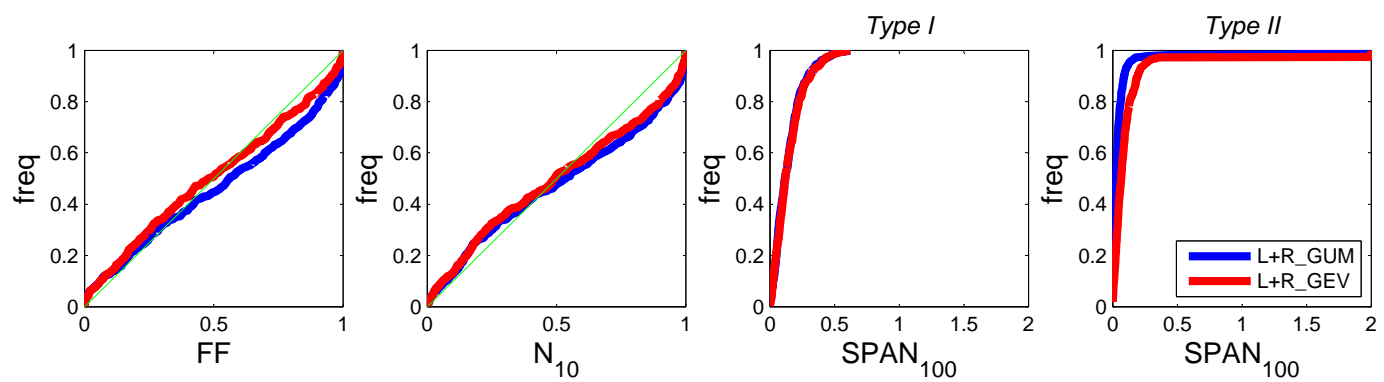

Fig. 5. Reliability $\left(F F, N_{10}\right)$ and stability $\left(\mathrm{SPAN}_{100}\right.$ - type I, SPAN 100 - type II) indices for local-regional implementations.
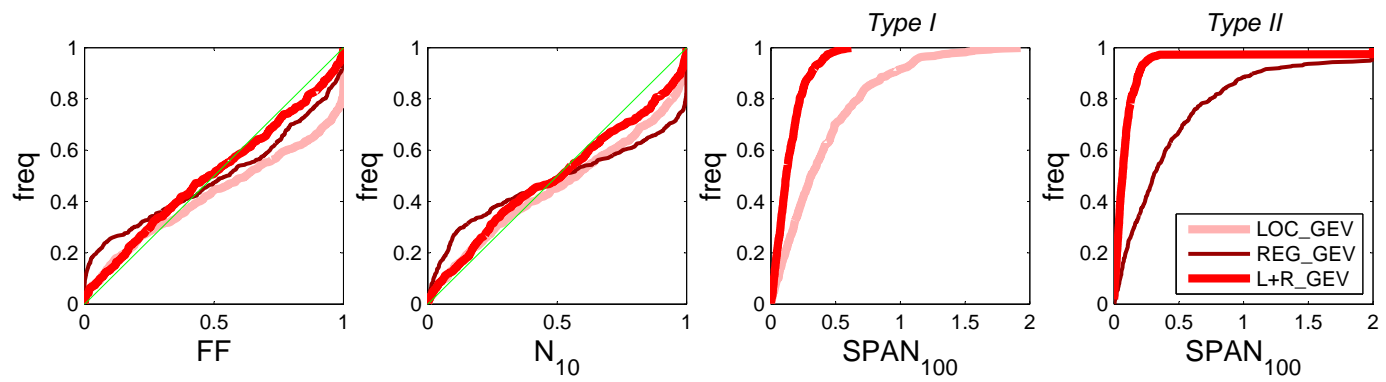

Fig. 6. Reliability $\left(F F, N_{10}\right)$ and stability $\left(\mathrm{SPAN}_{100}\right.$ - type I, SPAN 100 - type II) indices for local, regional and local-regional estimation of a GEV distribution.

two opponents for both indices $F F$ and $N_{10}$. In terms of stability, implementation L+R_GEV appears much more stable than both its purely local counterpart (type I stability) and its purely regional counterpart (Type II stability). These observations confirm that the GEV distribution is a sensible candidate for FFA, but that reliably estimating this distribution requires using both local and regional information.

\subsection{Stratification by region}

The results were presented so far at the scale of the whole country. However, Sect. 3.1 suggested that the differences between some implementations followed specific regional patterns. Figure 7 therefore shows reliability indices for Mediterranean (top, corresponding to regions 6 and 8 in Fig. 2) and Oceanic (bottom, regions 9, 10, 12 and 13 in Fig. 2) catchments. For readability, only implementations LOC_SHY, L+R_GUM and L+R_GEV (which appear to be the most reliable ones) are presented.

For Mediterranean catchments, the use of a Gumbel distribution (L+R_GUM) consistently yields below-diagonal reliability curves, denoting a tendency to underestimate quantiles. On the other hand, both implementations LOC_SHY and L+R_GEV yield acceptably similar reliability diagnostics. A tendency to slightly over-estimate large quantiles (indices $F F$ and $N_{100}$ ) might be suspected for LOC_SHY.

For Oceanic catchments, all three implementations yield similar results, suggesting that the evidence for rejecting the Gumbel distribution is weak in this region. We note however that using a GEV distribution does not deteriorate reliability (as long as it is estimated with a local-regional approach), and might therefore be preferred to the Gumbel distribution for its larger flexibility.

Lastly, a note of caution is made for this figure regarding the indices $N_{10}$ and $N_{100}$. It might appear surprising at first sight that curves are closer from the diagonal for $N_{100}$ than for $N_{10}$. However, this does not suggest that estimates of the 100-year flood are more reliable than estimates of the 10-year flood. Indeed, while comparing implementations for a given reliability index makes complete sense, a comparison of reliability indices for a given implementation is not meaningful, because the power to detect non-reliability strongly varies from index to index. In this particular case, curves appear closer from the diagonal for $N_{100}$ mostly because detecting failures in the estimation of the 100-year flood is much more challenging than for the 10-year flood, given the available sample size.

\subsection{Summary for all implementations}

Results for all implementations can be summarized by means of the reliability and stability scores described in Sect. 2.3.4. Figure 8 shows these scores on a radar plot, which confirms that the best overall competitors are LOC_SHY and L+R_GEV. LOC_SHY yields the highest stability scores, and the highest reliability scores for indices $F F$ and $N_{100}$. L+R_GEV has a higher score for index $N_{10}$, and might be slightly more reliable than LOC_SHY for Mediterranean 

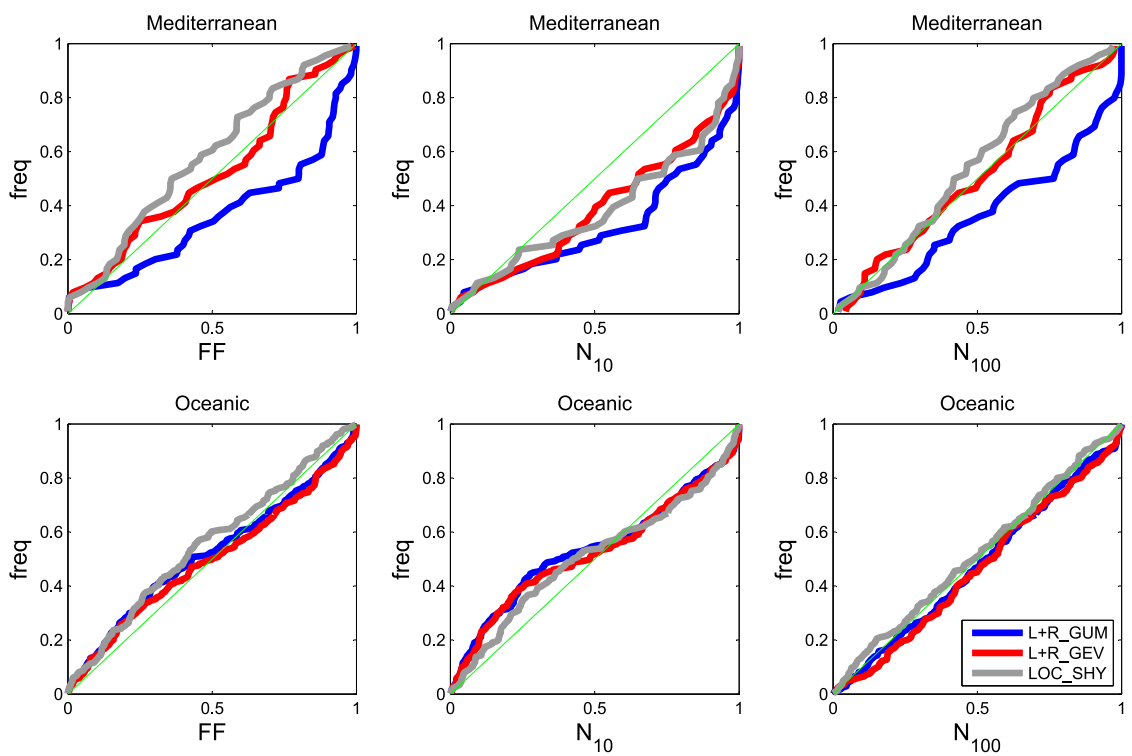

Fig. 7. Reliability indices for Mediterranean (top) and Oceanic (bottom) catchments.

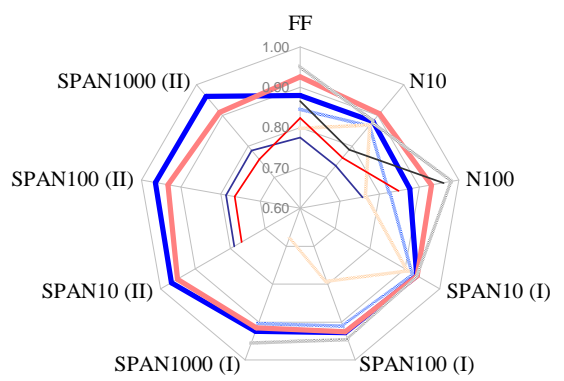

Fig. 8. Summary of reliability and stability scores for all implementations.

catchments. Note that implementation L+R_GUM is not considered as a finalist because, despite its excellent stability, it is systematically less reliable than LOC_SHY or L+R_GEV, and has been shown to be inadequate in the Mediterranean region (Sect. 3.5).

\section{Discussion}

\subsection{Results for different local sample sizes}

The results presented in this paper are conditional on the particular decompositions that were set up for stability and reliability assessments, and more precisely, on the sample size of 20 years for local data. One may therefore question whether the main findings of this study would still hold with different sample sizes.

Figure 9 indicates that the performances of the localregional implementation L+R_GEV and of LOC_SHY are not very sensitive to the local sample size. On the other hand, the performances of the local implementation LOC_GEV strongly deteriorate when the local sample size decreases. This indicates that the general conclusions summarized in Sect. 3.6 hold even more markedly with short samples.

Unfortunately, evaluating how performances evolve with larger samples is more challenging within this data-based comparison framework: indeed, the available series are not long enough to implement insightful calibration-validation decompositions with, e.g., 40 years used for calibration. The performance of local implementations is likely to improve with largest calibration samples. However, whether or not this would suffice to bridge the gap with the best implementations (L+R_GEV and LOC_SHY) remains to be seen. Monte Carlo experiments suggest that estimation errors can remain quite large even with "long" series of 40-50 years (not shown). This suggests that the benefit of complementing local data with either regional information (L+R_GEV) or information on the rainfall-runoff relationship (LOC_SHY), as advocated by Merz and Blöschl (2008a, b) and Viglione et al. (2013), may well remain significant with larger samples.

\subsection{Comparison with literature results}

Some results described in this paper have already been partly reported in the literature. In particular, the difficulty to locally estimate a GEV distribution with relatively short samples has been demonstrated through Monte Carlo experiments (e.g., Martins and Stedinger, 2000). The data-based comparison exercise described in this paper indicates that the resulting estimation errors lead to poor predictive performances that can also be demonstrated on real data. 

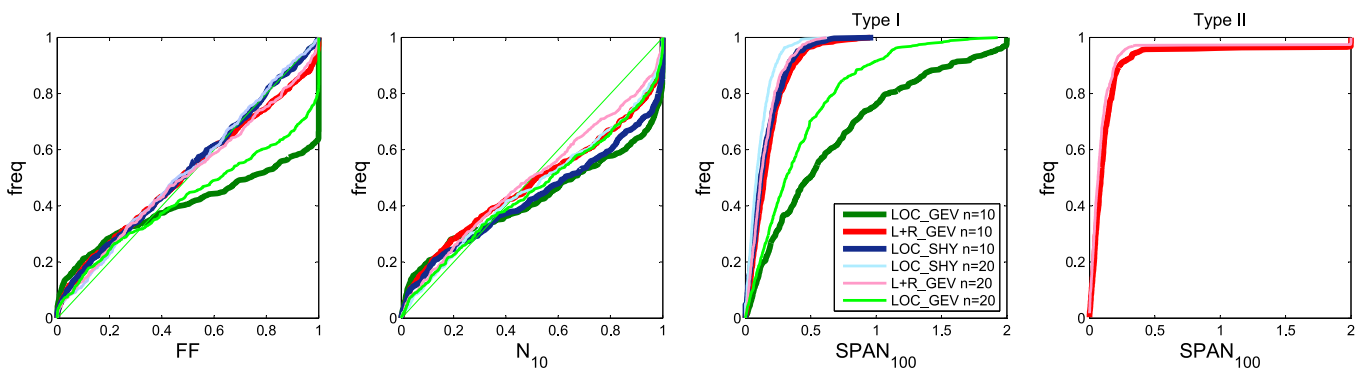

Fig. 9. Reliability $\left(F F, N_{10}\right)$ and stability $\left(\mathrm{SPAN}_{100}\right.$ - type I, SPAN 100 - type II) indices for three implementations, using 10 or 20 years of local data for calibration.

The main original results brought by this study are related to the comparison between distinct families of FFA implementations, including two distinct paradigms (estimation of a pre-specified distribution vs. continuous simulation) and several estimation scales (local, regional, local-regional). Such between-family comparisons are much more scarce in the literature. As far as we know, there have been no studies that reported Monte Carlo investigations to compare such distinct families, which is most probably due to the difficulty in setting up a fair Monte Carlo experiment, as explained in the introduction. Some authors compared the estimates arising from distinct families (e.g., Neppel et al., 2007), but they restricted the description to the differences between families, as opposed to ranking them according to their predictive performances. The evaluation carried out in this paper moves one step further by assessing and comparing predictive performances, which is necessarily a data-based exercise. For instance, the fact that the continuous simulation implementation LOC_SHY yields reliable predictions could not have been convincingly demonstrated using Monte Carlo simulations only (see discussion in Sect. 2.1).

Another result worth mentioning is the demonstrated inadequacy of the Gumbel distribution in Mediterranean catchments. The choice between a light-tailed Gumbel distribution and a heavy-tailed GEV distribution has been the subject of important debates in the literature: recently, global analyses were performed by Papalexiou and Koutsoyiannis (2013) and Serinaldi and Kilsby (2014) to assess this issue for extreme daily precipitations. Overall, at-site estimation suggests a preferentially heavy-tailed behavior of extreme rainfall at the global scale. The approach proposed in this paper might complement these analyses by evaluating whether using a light-tailed distribution leads to some demonstrable predictive failure in some regions of the world. Moreover, a joint assessment of the extremal behavior of both precipitation and streamflow at the global scale would also be of great interest.

\subsection{Limitations of the comparison framework}

While the comparison framework yielded valuable insights on the relative merits of distinct implementations, it is still affected by several limitations that are discussed here. Firstly, the ability to detect predictive failures for large quantiles is restricted by the length of available data. With the typical sample sizes (40-100 years), demonstrating a prediction failure for a 1000- or 10000-year quantile (which are of interest for risky structures such as dams or nuclear plants, for instance) is affected by huge uncertainty (see also Klemeš, 2000 and Serinaldi, 2013). In this paper, we focus on the 10 to 100 -year range. We do not consider floods of larger return period (i.e., > 100 years), since the data-based comparison framework is not powerful enough to draw conclusions for such large quantiles. It is therefore unclear whether the good performances of some implementations (LOC_SHY and L+R_GEV), as evaluated with limited sample sizes, still hold for extreme quantiles. On the other hand, the implementations showing poor performances have no reason to become highly capable when extrapolated to extreme quantiles, and can, therefore, be discarded.

A second limitation is related to the graphical nature of the comparison between implementations. It would be beneficial to implement a more quantitative comparison, e.g., based on hypothesis testing. For instance, it would be tempting to add significance limits around the diagonal in reliability plots (e.g., Fig. 4), as suggested by, e.g., Laio and Tamea (2007) based on a Kolmogorov-Smirnov test. Unfortunately, this cannot be done here because the test assumes independent data, but the values taken by the reliability indices are not fully independent from site to site (due to the spatial dependence existing between series from nearby sites).

Another limitation is that the comparison framework only produces global performance diagnostics, computed over a large number of sites. As a consequence, one should keep in mind that an implementation with excellent global performance may still fail on one or a few particular sites, without such isolated failures being detected by the global performance diagnostics.

Lastly, we stress that the comparison described in this paper was carried out with daily runoff (whose availability is much better than runoff recorded at a shorter time step). The good performance of some implementations (LOC_SHY and L+R_GEV) remains to be confirmed for shorter time steps, 
which are also of primary interest in engineering practice (e.g., flood peak estimation for flood design).

\section{Conclusions}

The objective of this paper was to report the results of a national comparison of the main FFA approaches used in France. This comparison was performed within a data-based framework, which enabled a direct assessment of the predictive performances of candidate FFA approaches. The main conclusions that can be drawn from the work can be summarized in the following points:

1. Two approaches, namely the local version of SHYREG and the local-regional estimation of a GEV distribution, seem to provide generally satisfactory results in terms of reliability and stability. The differences between the quantiles estimated by these two approaches are technically moderate.

2. In general, a local-regional estimation approach yields at least as good performances as its purely local or regional counterpart, and in some cases, it even clearly outperforms both of them.

3. In the oceanic-influenced catchments, the use of a Gumbel distribution seems acceptable. Local estimates yield relatively good performance indices. However the use of either the Gumbel or the GEV distribution within the mixed local-regional estimation approach results in similar or slightly improved reliability and stability indices.

4. In the Mediterranean area, we would not recommend using the Gumbel distribution, because it demonstrably underestimates quantiles. However, the local estimation of a GEV distribution is not recommended either, because the difficulty in estimating the shape parameter results in a clear lack of reliability. Therefore we recommend using LOC_SHY or local-regional mixed procedure for estimating the GEV-based quantiles in this area.

5. Estimation of flood quantiles in ungauged catchments remains a genuine challenge: all competing regional approaches evaluated in this work lead to a quite low reliability.

These main results suggest several avenues for future work. First, improving the purely regional FFA implementations appears to be a priority, given their quite low reliability. This requires improving the regression linking model parameters with catchment descriptors. A first possibility to achieve this improvement may be to include other descriptors. Alternative strategies include moving from fixed regions to a region-ofinfluence approach (e.g., Burn, 1990; Haddad and Rahman,
2012), or using specialized geostatistical methods to transfer information along the hydrologic network (e.g., Gottschalk, 1993; Sauquet, 2006; Skøien et al., 2006; Laaha et al., 2014).

In addition, combining several implementations may also yield further improvement. In particular, combining the bestperforming implementations LOC_SHY and L+R_GEV would make use of both regional and rainfall information to complement local discharge data. This would correspond to the spatial and causal expansion of information recently advocated by Merz and Blöschl (2008a, b), and implemented in a Bayesian framework by Viglione et al. (2013).

\section{Appendix A}

\section{Local implementations}

The PDF and CDF of the Gumbel distribution are

$f(x)=\frac{1}{\lambda} \exp \left(-\frac{x-\mu}{\lambda}-\exp \left(-\frac{x-\mu}{\lambda}\right)\right)$

$F(x)=\exp \left[-\exp \left(-\frac{x-\mu}{\lambda}\right)\right]$

$\lambda>0$,

where $\mu$ and $\lambda$ are the location and the scale parameters.

The PDF and CDF of the GEV distribution are

$$
\begin{aligned}
& f(x)=\frac{1}{\lambda}\left(1-\frac{\xi(x-\mu)}{\lambda}\right)^{\frac{1}{\xi}-1} \exp \left(-\left(1-\frac{\xi(x-\mu)}{\lambda}\right)^{\frac{1}{\xi}}\right) \\
& F(x)=\exp \left(-\left(1-\frac{\xi(x-\mu)}{\lambda}\right)^{\frac{1}{\xi}}\right) \\
& \lambda>0, \xi \neq 0,1-\frac{\xi(x-\mu)}{\lambda}>0,
\end{aligned}
$$

where $\mu, \lambda$ and $\xi$ are the location, scale and shape parameters.

Note that three families of distributions can be obtained depending on the value of the shape distribution: the Frechet family ( $\xi<0$, left-bounded distribution), the Weibull family $(\xi>0$, right-bounded distribution) and the Gumbel family ( $\xi \rightarrow 0$, unbounded distribution).

\section{A1 Regional implementations}

The regional estimation of Gumbel and GEV distributions uses a regression to link locally estimated parameters with catchment characteristics. Let $\theta_{i}$ denote the locally estimated location or scale parameter at site $i, \sigma_{i}$ denote its estimation standard deviation (i.e., the posterior standard deviation in this Bayesian context), and $x_{i}^{(1)}, \ldots, x_{i}^{\left(N_{\text {cov }}\right)}$ denote a set of $N_{\text {cov }}$ catchment characteristics used as covariates. The regression model for location and scale parameters can be written as follows:

$\log \left(\theta_{i}\right)=\beta_{0}+\sum_{j=1}^{N_{\text {cov }}} \beta_{j} x_{i}^{(j)}+\varepsilon_{i}, \varepsilon_{i} \sim N\left(0, \sqrt{\sigma_{\varepsilon}^{2}+\sigma_{i}^{2}}\right)$.

For the GEV distribution, an additional regression is required for the shape parameter. Since no significant 

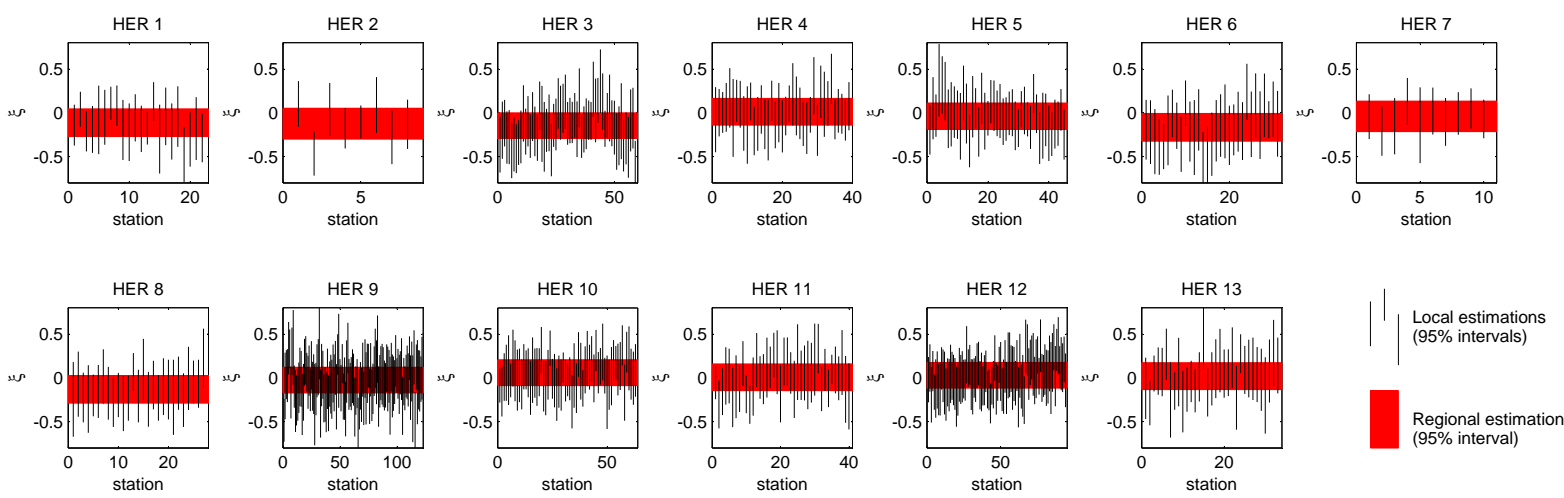

Fig. A1. Comparison of local and regional estimates of the shape parameter of a GEV distribution in each of the 13 hydroecoregions (HER).

relationship with catchment characteristics could be found, a constant regression is specified as follows:

$\xi_{i}=\beta_{0}+\varepsilon_{i}, \varepsilon_{i} \sim N\left(0, \sqrt{\sigma_{\varepsilon}^{2}+\sigma_{i}^{2}}\right)$.

Catchment characteristics are selected following Cipriani et al. (2012): (i) catchment area; (ii) mean elevation; (iii) mean 10-year rainfall (as given by Benichou and Le Breton, 1987); (iv) mean IDPR index (Index of Development and Persistence of the River networks (Mardhel et al., 2004), used as a proxy for the infiltration capacity). Moreover, regressions are estimated separately for each of the hydroecoregions shown in Fig. 2. Note that all four predictors are systematically used for all regions. A Bayesian estimation is used (with flat priors on $\left(\beta_{0}, \ldots, \beta_{N_{\text {cov }}}, \log \left(\sigma_{\varepsilon}\right)\right)$ ).

Note that Eq. (A4) effectively assumes that the shape parameter remains constant in each of the 13 hydroecoregions shown in Fig. 2. Empirical investigations suggest that this assumption is reasonable. As an illustration, Fig. A1 compares the local and regional estimates of the shape parameter: given estimation uncertainties, there is no strong evidence to reject the hypothesis of a constant shape parameter.

\section{A2 Local-regional implementations}

In local-regional implementations, a regional estimation is first applied to derive a prior distribution. At a given site $i$, the prior distribution of the location parameter is given by $\log \left(\mu_{i}\right) \sim N\left(\hat{\mu}_{i}, \hat{\sigma}_{\varepsilon}^{(\mu)}\right) . \hat{\mu}_{i}$ is computed by applying the regression in Eq. (A3), i.e., $\hat{\mu}_{i}=\exp \left(\hat{\beta}_{0}+\sum_{j=1}^{N_{\text {cov }}} \hat{\beta}_{j} x_{i}^{(j)}\right)$, and $\hat{\sigma}_{\varepsilon}^{(\mu)}$ is the estimated standard deviation of regression errors. Similarly, priors for the scale and shape parameters are given by

$$
\begin{aligned}
& \text { - Scale: } \log \left(\lambda_{i}\right) \sim N\left(\hat{\lambda}_{i}, \hat{\sigma}_{\varepsilon}^{(\lambda)}\right) \\
& \text { - GEV-only Shape: } \xi_{i} \sim N\left(\hat{\xi}_{i}, \hat{\sigma}_{\varepsilon}^{(\xi)}\right)
\end{aligned}
$$

At-site data are then used to compute the likelihood, and the posterior distribution therefore combines local and regional information.

Acknowledgements. This work was funded by the French Research Agency (ANR-08-RISKNAT-003) through the project ExtraFlo 2009-2013 (http://extraflo.irstea.fr/) and the FloodFreq COST Action ES0901 "European procedures for flood frequency estimation". We thank Francesco Serinaldi and John England for their very helpful comments and Claire Lauvernet for her invaluable technical assistance.

Edited by: T. Kjeldsen

Reviewed by: F. Serinaldi and J. England

\section{References}

Arnaud, P. and Lavabre, J.: Using a stochastic model for generating hourly hyetographs to study extreme rainfalls, Hydrol. Sci. J., 44, 433-446, 1999.

Arnaud, P. and Lavabre, J.: Coupled rainfall model and discharge model for flood frequency estimation, Water Resour. Res., 38, 11-1-11-11, doi:10.1029/2001WR000474, 2002.

Arnaud, P., Lavabre, J., Sol, B., and Desouches, C.: Regionalization of an hourly rainfall-generating model over metropolitan France for flood hazard estimation, Hydrol. Sci. J., 53, 34-47, 2008.

Aubert, Y.: Estimation des valeurs extrêmes de débit par la méthode Shyreg: Réflexions sur l'équifinalité dans la modélisation de la transformation pluie en débit, Pierre and Marie Curie University, Irstea Aix-en-Provence, 316 pp., 2013.

Benichou, P. and Le Breton, O.: Prise en compte de la topographie pour la cartographie des champs pluviométriques statistiques, La Météorologie, 7, 23-34, 1987.

Bobee, B., Cavadias, G., Ashkar, F., Bernier, J., and Rasmussen, P.: Towards a Systematic-Approach to Comparing Distributions Used in Flood Frequency-Analysis, J. Hydrol., 142, 121-136, 1993.

Burn, D. H.: Evaluation of regional flood frequency analysis with a region of influence approach, Water Resour. Res., 26, 22572265, doi:10.1029/WR026i010p02257, 1990. 
Carreau, J., Neppel, L., Arnaud, P. and Cantet, P.: Extreme rainfall analysis at ungauged sites in the South of France: comparison of three approaches, Journal de la Société Française de Statistique, 154, 119-138, 2013.

Cayla, O.: Probability calculation of design floods and inflows SPEED, Waterpower 1995, San Francisco, USA, 1995.

Cipriani, T., Toilliez, T., and Sauquet, E.: Estimating 10 year return period peak flows and flood durations at ungauged locations in France, La houille blanche, 2012.

England, J. F., Jarrett, R. D., and Salas, J. D.: Data-based comparisons of moments estimators using historical and paleoflood data, J. Hydrol., 278, 172-196, 2003.

Garavaglia, F., Lang, M., Paquet, E., Gailhard, J., Garçon, R., and Renard, B.: Reliability and robustness of rainfall compound distribution model based on weather pattern sub-sampling, Hydrol. Earth Syst. Sci., 15, 519-532, doi:10.5194/hess-15-519-2011, 2011.

Gottschalk, L: Correlation and covariance of runoff, Stochastic Hydrology and Hydraulics, 7, 85-101, 1993.

GREHYS: Inter-comparaison of regional flood frequency procedures for Canadian rivers., J. Hydrol., 186, 85-103, 1996.

Gunasekara, T. A. G. and Cunnane, C.: Split Sampling Technique for Selecting a Flood Frequency-Analysis Procedure, J. Hydrol., 130, 189-200, 1992.

Haddad, K. and Rahman, A: Regional flood frequency analysis in eastern Australia: Bayesian GLS regression-based methods within fixed region and ROI framework - Quantile Regression vs. Parameter Regression Technique, J. Hydrol., 430-431, 142161, doi:10.1016/j.jhydrol.2012.02.012, 2012.

Hosking, J. R. M. and Wallis, J. R.: Regional Frequency Analysis: an approach based on L-Moments, Cambridge University Press, Cambridge, UK, 226 pp., 1997.

Hosking, J. R. M., Wallis, J. R., and Wood, E. F.: An appraisal of the regional flood frequency procedure in the UK flood studies report, Hydrol. Sci. J., 30, 85-109, 1985.

Institution of Engineers Australia: Australian Rainfall and Runoff, edited by: Pilgrim, D. H., Engineers Australia, 1987.

Interagency Advisory Committee on Water Data: Guidelines for determining flood-flow frequency: Bulletin 17B of the Hydrology Subcommittee, edited by: Coordination, O. o. W. D., US Geological Survey, Reston, Va., 1982.

Klemeš, V.: Tall tales about tails of hydrological distributions, II, J. Hydrol. Eng., 5, 232-239, doi:10.1061/(ASCE)10840699(2000)5:3(232), 2000.

Kochanek, K., Renard, B., Lang, M., and Arnaud, P.: Comparison of several at-site flood frequency models on a large set of French discharge series, The 2nd European Conference on FLOODrisk Management. Science Policy and Practice: Closing the Gap, Rotterdam, the Netherlands, 2012.

Kroll, C. N. and Stedinger, J. R.: Estimation of moments and quantiles using censored data, Water Resour. Res., 32, 1005-1012, 1996.

Kuczera, G.: Comprehensive at-site flood frequency analysis using Monte Carlo Bayesian inference, Water Resour. Res., 35, 15511557, 1999.

Laaha, G., Skøien, J. O., and Blöschl, G.: Spatial prediction on river networks: comparison of top-kriging with regional regression, Hydrol. Process., 28, 315-324, doi:10.1002/hyp.9578, 2014.
Laio, F. and Tamea, S.: Verification tools for probabilistic forecasts of continuous hydrological variables, Hydrol. Earth Syst. Sci., 11, 1267-1277, doi:10.5194/hess-11-1267-2007, 2007.

Lang, M., Lavabre, J., Sauquet, E., and Renard, B.: Guide méthodologique pour l'estimation de la crue centennale dans le cadre des plans de prévention des risques d'inondation, edited by: Quae, E., Editions Quae, 134 pp., 2007.

Mardhel, V., Frantar, P., Uhan, J., and Mio, A.: Index of development and persistence of the river networks as a component of regional groundwater vulnerability assessment in Slovenia, Int. Conf. groundwater vulnerability assessment and mapping, Ustron, Poland, 2004.

Martins, E. S. and Stedinger, J. R.: Generalized maximumlikelihood generalized extreme-value quantile estimators for hydrologic data, Water Resour. Res., 36, 737-744, 2000.

Merz, R. and Blöschl, G.: Flood frequency hydrology: 1. Temporal, spatial, and causal expansion of information, Water Resour. Res., 44, W08432, doi:10.1029/2007WR006744, 2008a.

Merz, R. and Blöschl, G.: Flood frequency hydrology: 2. Combining data evidence,Water Resour. Res., 44, W08433, doi:10.1029/2007WR006745, 2008b.

Meshgi, A. and Khalili, D.: Comprehensive evaluation of regional flood frequency analysis by L- and LH-moments. II. Development of LH-moments parameters for the generalized Pareto and generalized logistic distributions, Stoch. Environ. Res. Risk Assess., 23, 137-152, 2009.

Neppel, L., Arnaud, P., and Lavabre, J.: Extreme rainfall mapping: Comparison between two approaches in the Mediterranean area, C. R. Geosci., 339, 820-830, doi:10.1016/j.crte.2007.09.013, 2007.

Organde, D., Arnaud, P., Fine, J.-A., Fouchier, C., Folton, N., and Lavabre, J.: Régionalisation d'une méthode de prédétermination de crue sur l'ensemble du territoire français : la méthode SHYREG, Revue des sciences de l'eau, J. Water Sci., 26, 65-78, doi:10.7202/1014920ar, 2013.

Ouarda, T., Cunderlik, J. M., St-Hilaire, A., Barbet, M., Bruneau, P., and Bobee, B.: Data-based comparison of seasonality-based regional flood frequency methods, J. Hydrol., 330, 329-339, 2006.

Papalexiou, S. M. and Koutsoyiannis, D.: Battle of extreme value distributions: A global survey on extreme daily rainfall, Water Resour. Res., 49, doi:10.1029/2012WR012557, 2013.

Paquet, E., Gailhard, J., and Garcon, R.: Evolution de la méthode du gradex: approche par type de temps et modélisation hydrologique, La houille blanche, 5, 80-90, 2006.

Reed, D. W., Faulkner, D. S., Robson, A. J., Houghton-Carr, H., and Bayliss, A. C.: Flood Estimation Handbook, edited by: Institute of Hydrology, Wallingford, 1999.

Renard, B., Lang, M., Bois, P., Dupeyrat, A., Mestre, O., Niel, H., Sauquet, E., Prudhomme, C., Parey, S., Paquet, E., Neppel, L., and Gailhard, J.: Regional methods for trend detection: Assessing field significance and regional consistency, Water Resour. Res., 44, W08419, doi:10.1029/2007WR006268, 2008.

Renard, B., Kochanek, K., Lang, M., Garavaglia, F., Paquet, E., Neppel, L., Najib, K., Carreau, J., Arnaud, P., Aubert, Y., Borchi, F., Soubeyroux, J. M., Jourdain, S., Veysseire, J. M., Sauquet, E., Cipriani, T., and Auffray, A.: Data-based comparison of frequency analysis methods: A general framework, Water Resour. Res., 49, 825-843, doi:10.1002/wrcr.20087, 2013. 
Ribatet, M., Sauquet, E., Gresillon, J. M., and Ouarda, T. B. M. J.: A regional Bayesian POT model for flood frequency analysis, Stoch. Environ. Res. Risk Assess., 21, 327-339, 2006.

Sankarasubramanian, A. and Srinivasan, K.: Investigation and comparison of sampling properties of L-moments and conventional moments, J. Hydrol., 218, 13-34, 1999.

Sauquet, E: Mapping mean annual river discharges: Geostatistical developments for incorporating river network dependencies, J. Hydrol., 331, 300-314, doi:10.1016/j.jhydrol.2006.05.018, 2006.

Serinaldi, F.: An uncertain journey around the tails of multivariate hydrological distributions, Water Resour. Res., 49, 6527-6547, doi:10.1002/wrcr.20531, 2013.

Serinaldi, F. and Kilsby, C. G.: Rainfall extremes: Towards reconciliation after the battle of distributions, Water Resour. Res., 50, doi:10.1002/2013WR014211, 2014.

Skøien, J. O., Merz, R., and Blöschl, G.: Top-kriging - geostatistics on stream networks, Hydrol. Earth Syst. Sci., 10, 277-287, doi:10.5194/hess-10-277-2006, 2006.
Stedinger, J. R. and Tasker, G. D.: Regional hydrologic analysis: 1. Ordinary, weighted and generalized least squares compared, Water Resources Research, 21, 1421-1432 [Correction, Water Resour. Res., 1422, 1844, 1986.], 1985.

Stedinger, J. R. and Tasker, G. D.: Regional hydrologic analysis: 2. Model-error estimators, estimation of sigma and log-Pearson type 3 distributions, Water Resour. Res., 22, 1487-1499, 1986.

Stewart, E. J., Kjeldsen, T. R., Jones, D. A., and Morris, D. G.: The flood estimation handbook and UK practice:past, present and future, in: Flood Risk Management: Research and Practice, edited by: Samuels, P., Huntington, S., Allsop, W., and Harrop, J., CRC Press, 179, 2008.

Viglione, A., Merz, R., Salinas, J. L., and Blöschl, G.: Flood frequency hydrology: 3. A Bayesian analysis, Water Resour. Res., 49, 675-692, doi:10.1029/2011WR010782, 2013.

Wasson, J. G., Chandesris, A., Pella, H., and Blanc, L.: Les hydroécorégions: une approche fonctionnelle de la typologie des rivières pour la directive cadre européenne sur l'eau, Ingénieries, 40, 3-10, 2004. 